PROCEEDINGS OF THE

AMERICAN MATHEMATICAL SOCIETY

Volume 128, Number 7, Pages 2147-2154

S 0002-9939(99)05277-6

Article electronically published on October 29, 1999

\title{
EMBEDDED SURFACES AND ALMOST COMPLEX STRUCTURES
}

\author{
CHRISTIAN BOHR
}

(Communicated by Ronald A. Fintushel)

\begin{abstract}
In this paper, we prove necessary and sufficient conditions for a smooth surface in a smooth 4-manifold $X$ to be pseudoholomorphic with respect to an almost complex structure on $X$. In particular, this provides a systematic approach to the construction of pseudoholomorphic curves that do not minimize the genus in their homology class.
\end{abstract}

\section{INTRODUCTION AND SUMMARY OF RESULTS}

Let $X$ be a closed differentiable and connected 4-manifold with an orientation and $\Sigma \subset X$ a connected oriented surface. An almost complex structure $\mathbf{J}$ on $X$ is a reduction of the structure group $\mathrm{GL}^{+}(4)$ of $T X$ to the group $\mathrm{GL}(2, \mathbb{C})$, or, in other words, a section $J$ of $\operatorname{End}(T X)$ with $J^{2}=-1$ that preserves the orientation, so that $T X$ carries the structure of a complex vector bundle. The surface $\Sigma$ is called a pseudoholomorphic curve if the tangent bundle of $\Sigma$ is preserved by $J$ (note that in this case, the almost complex structure on $X$ induces a complex structure on $\Sigma$ ). The question that shall be treated on the following pages is: Given a surface $\Sigma$, is there an almost complex structure $J$ on $X$ such that $\Sigma$ is a pseudoholomorphic curve with respect to $J$ ?

First, recall that an almost complex structure $J$ has a first Chern class $c_{1}(J) \in$ $H^{2}(X ; \mathbb{Z})$ (this is just the first Chern class of $T X$ considered as a complex vector bundle) and that this class has the properties

1. $c_{1}(J)^{2}=2 \chi+3 \tau$,

2. $c_{1}(J) \equiv w_{2} \bmod 2$,

where $\chi$ denotes the Euler characteristic and $\tau$ the signature of the intersection form of $X$. A class with property 2 is called a characteristic class on $X$. If the homology of $X$ does not contain 2-torsion, then these classes can be characterized in terms of the intersection form $Q$ of $X$ : a class $c \in H^{2}(X ; \mathbb{Z})$ is characteristic if and only if $Q(x, x) \equiv Q(x, c) \bmod 2$ for all $x \in H^{2}(X ; \mathbb{Z})$. If there is 2-torsion, one part of this statement is still true: if $c$ is characteristic, then $Q(x, c) \equiv Q(x, x) \bmod 2$ for every $x$. Conversely, if a class $c$ fulfills $Q(x, c) \equiv Q(x, x) \bmod 2$ for all $x$, then there is a torsion class $a$ such that $c+a \equiv w_{2}(X) \bmod 2$. It is a classical result of Whitney that there are characteristic classes on any 4-manifold ([W]).

Received by the editors August 17, 1998.

1991 Mathematics Subject Classification. Primary 53C15, 57R95.

This work has been supported by the Graduiertenkolleg "Mathematik im Bereich ihrer Wechselwirkung mit der Physik".

(C)2000 American Mathematical Society 
Furthermore, it is well known that in turn every class in $H^{2}(X ; \mathbb{Z})$ fulfilling the conditions 1 and 2 can be realized as the first Chern class of an almost complex structure. So there is an almost complex structure on $X$ if and only if there is a class in $H^{2}(X ; \mathbb{Z})$ that fulfills the conditions above (this is a result of $\mathrm{Wu}$, see $[\mathrm{HH}]$ ). In fact, every such class is the first Chern class of an almost complex structure. Consideration of the intersection form easily leads to the conclusion that if the intersection form of $X$ is indefinite, there is an almost complex structure on $X$ if and only if $b_{1}+b_{2}^{+}$is odd, where $b_{2}^{+}$denotes the maximal dimension of a subspace of $H^{2}(X, \mathbb{R})$ on which the intersection form is positive definite.

Definition 1. Let $G$ be a finitely generated abelian group and $g \in G$. Let $T \subset G$ be the torsion subgroup of $G$.

1. If $G$ is free abelian and $g \neq 0$, the divisibility of $g$ in $G$ is defined to be the largest positive integer $d$ with the property that there is an $x \in G$ with $g=d x$. The divisibility of $0 \in G$ is defined to be zero.

2. For arbitrary $G$, the divisibility $d(g)$ of $g \in G$ is the divisibility of $g$ (more precisely, the residue class of $g$ ) in the free abelian group $G / T$. The divisibility is defined to be zero if and only if $g \in T$.

Remark 1. Clearly the image of the homomorphism $\operatorname{Hom}(G ; \mathbb{Z}) \rightarrow \mathbb{Z}$, given by evaluation on $g$, is just $d(g) \mathbb{Z}$. From this, we see that the divisibility of $k \cdot g$ for $g \in G, k \in \mathbb{Z}$ is $\pm k$ times the divisibility of $g$.

Definition 2. Let $(\Gamma, Q)$ be a lattice (i.e. $\Gamma$ is a free abelian group of finite rank and $Q$ a unimodular symmetric bilinear form on $\Gamma$ ). For $\gamma \in \Gamma$ with divisibility $d=d(\gamma)$ define $k(\gamma) \in \mathbb{Z}_{d}$ as follows: Choose a characteristic class $c \in \Gamma$, i.e. $Q(c, x) \equiv Q(x, x) \bmod 2$ for every $x \in \Gamma$, and set

$$
k(\gamma):=1+\frac{1}{2}(Q(\gamma, \gamma)-Q(c, \gamma)) \bmod d .
$$

The residue class $k(\gamma)$ is independent of the choice of $c$ : if $c^{\prime}$ is another characteristic class, then $c$ and $c^{\prime}$ differ by a multiple of 2 , so the terms in the bracket differ by a multiple of $2 d$, according to Remark 1 and this does not affect $k(\gamma)$. If $\gamma=0$, then $k(\gamma)=1 \in \mathbb{Z}$.

Definition 3. For a closed connected and oriented surface $\Sigma \subset X$ let $k(\Sigma)=$ $k([\Sigma])$ with respect to the lattice defined by the intersection form on the free group $H_{2}(X ; \mathbb{Z}) /$ Tor $H_{2}(X ; \mathbb{Z})$, where $[\Sigma]$ denotes the homology class of $\Sigma$.

Since cup products are not altered by adding torsion classes to one of the factors, we could as well have defined $k([\Sigma])$ by

$$
k([\Sigma])=1+\frac{1}{2}(\Sigma \cdot \Sigma-c \cdot \Sigma) \bmod d
$$

where $d$ denotes the divisibility of $[\Sigma]$ in $H_{2}(X ; \mathbb{Z})$ and $c$ is any characteristic class on $X$, i.e. $c \equiv w_{2}(X) \bmod 2$. We will use the notation $d(\Sigma)$ for the divisibility of the class $[\Sigma]$.

If there is an almost complex structure $J$ turning $\Sigma$ into a pseudoholomorphic curve, then the adjunction formula

$$
g(\Sigma)=1+\frac{1}{2}\left(\Sigma \cdot \Sigma-c_{1}(J) \cdot \Sigma\right)
$$


holds, and $c_{1}(J)$ is characteristic, so we have the congruence

$$
g(\Sigma) \equiv k(\Sigma) \bmod d
$$

It turns out that this necessary condition is in fact sufficient for the existence of such a $J$ if the intersection form of $X$ is strictly indefinite (i.e. $\min \left\{b_{2}^{+}, b_{2}^{-}\right\} \geq 2$ ):

Theorem 1. Let $X$ be a connected oriented closed and differentiable 4-manifold and $\Sigma \subset X$ a closed connected and oriented surface with divisibility d. Suppose $\min \left\{b_{2}^{+}, b_{2}^{-}\right\} \geq 2$ and $b_{1}+b_{2}^{+} \equiv 1 \bmod 2$. Then there is an almost complex structure $J$ on $X$ such that the surface $\Sigma$ is pseudoholomorphic with respect to $J$ if and only if $g(\Sigma) \equiv k(\Sigma) \bmod d$.

Note that this condition is in particular fulfilled when the class of $\Sigma$ has divisibility one, so any such surface is pseudoholomorphic with respect to an almost complex structure on $X$. In addition, if $[\Sigma]$ is not a torsion class, this condition is "cyclic": we can attach handles that do not change the homology class of $\Sigma$ - and hence do not alter $k(\Sigma)$ - but raise the genus until the condition of the theorem is fulfilled. In this way we even can produce a surface $\Sigma^{\prime}$ homologous to $\Sigma$ that is pseudoholomorphic with respect to an almost complex structure on $X$, but whose genus is arbitrarily large:

Corollary 1. Let $X$ be a closed connected and oriented smooth 4-manifold with $b_{1}+b_{2}^{+} \equiv 1 \bmod 2$ and $\min \left\{b_{2}^{+}, b_{2}^{-}\right\} \geq 2$, and let $\Sigma$ be a surface in $X$ such that $[\Sigma]$ is not a torsion class. Let $m \in \mathbb{N}$ be any natural number. Then there is an almost complex structure $J$ on $X$ and a pseudoholomorphic curve $\Sigma^{\prime}$ homologous to $\Sigma$ with $g\left(\Sigma^{\prime}\right) \geq g(\Sigma)+m$.

This corollary provides a large number of pseudoholomorphic curves that do not minimize the genus in their homology class. Other examples for this have been given by Kotschick (unpublished) and in a paper by Mikhalkin (Mi]). Although the case $X=\mathbb{C} P^{2}$ is not covered by the corollary, this can also occur on $\mathbb{C} P^{2}$, an example for this is the curve in the statement of Proposition 4.

The next three propositions show that the condition $\min \left\{b_{2}^{+}, b_{2}^{-}\right\} \geq 2$ is really necessary; if it is dropped, the theorem is no longer true:

Proposition 1. If $X$ is a rational complex surface, there is a surface $\Sigma$ in $X$ with $g(\Sigma) \equiv k(\Sigma) \bmod d(\Sigma)$ that is not pseudoholomorphic with respect to any almost complex structure on $X$.

Proposition 2. Let $X$ be a 4-manifold with definite intersection form. Then there is a surface $\Sigma$ in $X$ with $k(\Sigma) \equiv g(\Sigma) \bmod d(\Sigma)$ that is not pseudoholomorphic with respect to any almost complex structure on $X$.

Proposition 3. Let $Q$ be a unimodular symmetric bilinear form over $\mathbb{Z}$ fulfilling $\min \left\{b^{+}, b^{-}\right\} \leq 1$ that can be realized as the intersection form of a smooth 4-manifold. In the case that $Q$ is indefinite and even assume that the signature of $Q$ is non-negative. Then there is a closed oriented 4-manifold $X$ having $Q$ as intersection form and a closed oriented and connected surface $\Sigma \subset X$ such that $b_{1}(X)+b_{2}^{+}(X) \equiv 1 \bmod 2$ and $g(\Sigma) \equiv k(\Sigma) \bmod d(\Sigma)$, but $\Sigma$ is not pseudoholomorphic with respect to any almost complex structure on $X$.

Note that all odd forms and all even intersection forms of smooth 4-manifolds that have no 2-torsion in their homology $([\mathrm{D}])$ or are spin $([\mathrm{Fu}])$ are covered by this proposition. 
Finally, there is a simple example for the case $X=\mathbb{C} P^{2}$. The class $-1 \in$ $H_{2}\left(\mathbb{C} P^{2} ; \mathbb{Z}\right)$ can be represented by a sphere - just take the complex line with the orientation reversed - hence the minimal genus for this class is 0 . The following proposition therefore provides another example that a pseudoholomorphic curve does not always minimize the genus in its homology class:

Proposition 4. There is a surface with genus 3, representing minus the generator of $\mathrm{H}_{2}\left(\mathbb{C} P^{2} ; \mathbb{Z}\right)$, that is pseudoholomorphic with respect to an almost complex structure homotopic to the canonical one.

\section{Proofs of Theorem 1 and Proposition 4}

For the proofs, we need two lemmas, the first of them being a topological lemma, whereas the second one is purely algebraic:

Lemma 1. If there is a class $c \in H^{2}(X ; \mathbb{Z})$ with the properties

1. $c^{2}=2 \chi+3 \tau$,

2. $c \equiv w_{2} \bmod 2$,

3. $\langle c,[\Sigma]\rangle=2-2 g(\Sigma)+\Sigma \cdot \Sigma$,

then there is an almost complex structure $J$ such that the surface $\Sigma$ is pseudoholomorphic with respect to $J$ and $c_{1}(J)=c$ (here $\tau$ denotes the signature of $X$ ).

Proof. By the result of $\mathrm{Wu}$ mentioned earlier (see $[\mathrm{HH}]$ ), there is an almost complex structure $J_{0}$ on $X$ with $c_{1}\left(J_{0}\right)=c$. Introduce a Riemannian metric $g$ on $X$ compatible with $J_{0}$, i.e. the endomorphism $J$ is isometric on the fibers of $T X$ with respect to $g$. Then the almost complex structures compatible with $g$ can be identified with the reductions of the structure group $\mathrm{SO}(4)$ to $\mathrm{U}(2)$, i.e. with sections in the bundle $\Theta:=P_{\mathrm{SO}(4)} / \mathrm{U}(2)$ having fiber $\mathrm{SO}(4) / \mathrm{U}(2)=S^{2}$. For the restriction of the tangent bundle to $\Sigma$, we have a decomposition $T X \mid \Sigma=N \oplus T \Sigma$, where $N$ denotes the normal bundle of $\Sigma$. By introducing metrics on these two bundles, their structure group can be reduced to $\mathrm{SO}(2)$. Since $\mathrm{SO}(2) \times \mathrm{SO}(2) \subset \mathrm{U}(2)$, we have an almost complex structure on $T X \mid \Sigma$ turning this decomposition into a direct sum of complex vector bundles. This almost complex structure can be extended to an almost complex structure $J$ on the disk bundle $D N$ (that is identified with a tubular neighborhood of $\Sigma$ ). Clearly, $\Sigma$ is a pseudoholomorphic curve in $D N$ with respect to $J$. We now have to show that $J$ can be extended over $X$ to an almost complex structure homotopic to $J_{0}$ as a section of $\Theta$. Then $c_{1}(J)=c_{1}\left(J_{0}\right)=c$, and the lemma is proved.

The second cohomology $H^{2}(D N ; \mathbb{Z})$ is $\mathbb{Z}$, generated by the fundamental class $[\Sigma]$ (more exactly, by its pullback via the projection $D N \rightarrow \Sigma$ ). Let $s$, respectively $s_{0}$, denote the sections of $\Theta$ on $D N$ given by $J$ and $J_{0}$. Note that $J_{0}$ defines an extension of $s_{0}$ to $X$. Let $c_{1} \in H^{2}(D N ; \mathbb{Z})$ denote the first Chern class of $J$. By definition of $J$, we have a decomposition $(T X \mid \Sigma, J)=N \oplus T \Sigma$ of complex vector bundles. Taking the first Chern class on both sides yields the adjunction equality $\left\langle c_{1}, \Sigma\right\rangle=2-2 g+\Sigma \cdot \Sigma$. But by assumption 3 , the same is true for $c=c_{1}\left(J_{0}\right)$, hence $c_{1}=c$ in $H^{2}(D N ; \mathbb{Z})$. A short calculation, using the exact homotopy sequence of the fibration

$$
S^{2} \rightarrow B \mathrm{U}(2) \rightarrow B \mathrm{SO}(4),
$$

yields that $\pi_{2}\left(S^{2}\right) \rightarrow \pi_{2}(B \mathrm{U}(2))$ is the multiplication by 2 , and this shows that for the primary difference $p \in H^{2}(D N ; \mathbb{Z})$ between $s$ and $s_{0}$ as sections $D N \rightarrow \Theta$, 
we have the equality $2 p=c_{1}-c=0$. Since the homology of $D N$ is torsion free, this implies $p=0$, and since $H^{3}(D N ; \mathbb{Z})=H^{4}(D N ; \mathbb{Z})=0$, there are no higher obstructions; hence the sections $s$ and $s_{0}$ are homotopic on $D N$. Using the homotopy extension property we can conclude that there is an extension of $s$ to $X$ homotopic to $s_{0}$, and this proves the assertion.

Lemma 2. Let $(\Gamma, Q)$ be a lattice with $\min \left\{b^{+}, b^{-}\right\} \geq 2$, let $\gamma \in \Gamma$ be a vector with divisibility $d$, let $h$ be an integer with $h \equiv \tau(Q) \bmod 8$, where $\tau(Q)$ denotes the signature of $Q$, and let $g$ be a natural number with $g \equiv k(\gamma) \bmod d$. Then there is $a c \in \Gamma$ with

1. $c$ is characteristic, i.e. $Q(c, x) \equiv Q(x, x) \bmod 2$ for every $x \in \Gamma$,

2. $Q(c, c)=h$ and

3. $Q(c, \gamma)=2-2 g+Q(\gamma, \gamma)$.

Proof. According to the classification theorem of Hasse-Minkowski (see $[\mathrm{MH}]$ ), we can choose a basis $\left(e_{1}, \ldots, e_{n}\right)$ such that with respect to this basis, $Q$ is described by the matrix

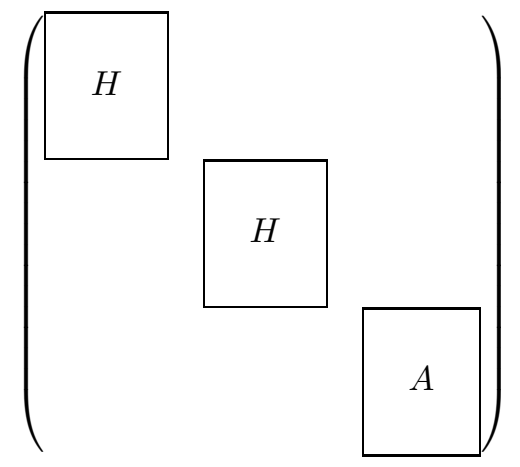

where $H=\left(\begin{array}{ll}0 & 1 \\ 1 & 0\end{array}\right)$ denotes the hyperbolic form, and $A$ is diagonal if $Q$ is odd, or of the type $m E_{8}$ with some $m \in \mathbb{Z}$ in the case that $Q$ is even. If $\gamma=0$, the condition on $g$ reads $g=1$, and any characteristic $c$ with $Q(c, c)=h$ will do the job (it is easy to see that such a $c$ exists). Now assume $\gamma \neq 0$. Let $\gamma=d p$ with $p \in \Gamma$ having divisibility one.

Case 1: $Q$ is even. Then $p$ must be ordinary (i.e. not characteristic), because $Q$ is unimodular and - according to the characterisation of the divisibility given in Remark 1- therefore there is an $x \in \Gamma$ with $Q(x, p)=1$. Using a result of Wall ([W1]) concerning the group of automorphisms of $Q$, we can assume $p=(k, 1,0, \ldots, 0)$ with some $k \in \mathbb{Z}$ (Wall's Theorem asserts that there is an automorphism that maps $p$ to some vector of this type, after a change of the basis, we can assume that $p$ has this special form). Let $c_{0} \in\left\langle e_{3}, \ldots, e_{n}\right\rangle$ be some characteristic vector with $Q\left(c_{0}, c_{0}\right)=h$ (it is easy to see that such a $c_{0}$ exists, using the Hasse-Minkowski classification applied to $H \oplus A)$. The assumption on $g$ implies that the difference between $Q\left(c_{0}, \gamma\right)$ and $2-2 g+Q(\gamma, \gamma)$ is a multiple of $2 d$, say $2 d a$ with $a \in \mathbb{Z}$. Let $c=c_{0}+2 a e_{1}$. Then $Q(c, c)=Q\left(c_{0}, c_{0}\right)=h$ and $Q(c, \gamma)=Q\left(c_{0}, \gamma\right)+2 a d=2_{2} g+Q(\gamma, \gamma)$.

Case 2: $Q$ is odd: a) $p$ is ordinary: Then, again using the result of Wall, we can assume that $p=\left(k, 1, p^{\prime}\right)$ with $p^{\prime} \in\left\langle e_{3}, \ldots, e_{n}\right\rangle$, and the same arguments as in Case 1 apply. 
b) $p$ is characteristic: Since $Q$ is odd, the same must be true for $A$, in particular, $n \geq 5$. In this case, the standard form for $p$ is $p=(0,0,2 k, 2,1, \ldots, 1)$ with some $k \in \mathbb{Z}$, because this vector is characteristic, has divisibility one and square $8 k+\tau(Q)$, and $Q(p, p) \equiv \tau(Q) \bmod 8$. Choose any characteristic vector $c_{0} \in\left\langle e_{3}, \ldots, e_{n}\right\rangle$. Then the difference $Q\left(c_{0}, c_{0}\right)-(2-2 g+Q(\gamma, \gamma))$ is divisible by $2 d$ (this follows from the assumption on $g$ and the definition of $k(\gamma)$ ). Therefore we can choose $a \in \mathbb{Z}$ such that $c_{1}:=c_{0}+2 a e_{5}$ has the properties 1 and 3 (observe $Q\left(e_{5}, p\right)= \pm 1$ ). The difference between $Q\left(c_{1}, c_{1}\right)$ and $h$ is now a multiple of 8 , say $8 b, b \in \mathbb{Z}$, and therefore $c=c_{1}+2 b e_{1}+2 e_{2}$ fulfills all three conditions.

Proof of Proposition 4 . Let $J$ denote the standard almost complex structure on $\mathbb{C} P^{2}$ with Chern class $c_{1}(J)=3$. Let $\Sigma^{\prime}$ denote the complex line $\mathbb{C} P^{1}$ in $\mathbb{C} P^{2}$ with the orientation reversed, hence $\left[\Sigma^{\prime}\right]=-1$. By attaching handles, we can construct a surface $\Sigma \subset \mathbb{C} P^{2}$ with genus 3 homologous to $\Sigma^{\prime}$. For this surface, we have

$$
\left\langle c_{1}(J),[\Sigma]\right\rangle=-3=2-2 g(\Sigma)+\Sigma \cdot \Sigma,
$$

and the assertion follows using the homotopy argument as in the proof of Lemma 1

Proof of Theorem 1 . First assume that $\Sigma$ is pseudoholomorphic with respect to $J$. Then we have the adjunction equality $c_{1}(J) \cdot \Sigma=2-2 g+\Sigma \cdot \Sigma$, and this implies $g(\Sigma) \equiv k(\Sigma) \bmod d$. For the converse, let $\Sigma$ fulfill $g(\Sigma)=k(\Sigma) \bmod d$. Let $\Gamma=H^{2}(X ; \mathbb{Z}) /$ Tor $H^{2}(X ; \mathbb{Z})$ and $Q$ denote the form on $\Gamma$ defined by the intersection form. Let $\gamma \in \Gamma$ be the residue class of $[\Sigma]$ and $h:=2 \chi+3 \tau$. A short calculation shows that the condition $b_{1}+b_{2}^{+} \equiv 1 \bmod 2$ implies that $\chi+\tau$ is divisible by 4 and $h \equiv \tau(Q) \bmod 8$. According to Lemma 2, there is a $c^{\prime} \in \Gamma$ with $Q\left(c^{\prime}, c^{\prime}\right)=h, Q\left(c^{\prime}, \gamma\right)=2-2 g+Q(\gamma, \gamma)$ and $Q\left(c^{\prime}, x\right) \equiv Q(x, x) \bmod 2$ for all $x \in \Gamma$. Choose a lift $c \in H^{2}(X ; \mathbb{Z})$ of $c^{\prime}$ such that $c \equiv w_{2}(X) \bmod 2$. Then $c$ fulfills the conditions of Lemma 1, and the assertion of the theorem follows.

\section{Proof of Propositions 1, 2 and 3}

Proof of Proposition 1. A rational surface is diffeomorphic to $S^{2} \times S^{2}$ or to $\mathbb{C} P^{2} \# k \overline{\mathbb{C} P^{2}}$. First, consider the case $X=S^{2} \times S^{2}$. Let $\Delta$ denote the diagonal sphere in $S^{2} \times S^{2}$. Whenever $c=(x, y) \in H^{2}(X ; \mathbb{Z})=\mathbb{Z} \times \mathbb{Z}$ is the Chern class of an almost complex structure, we have $c^{2}=2 x y=8$, and $x, y$ are even; therefore we have $c=(2,2)$ or $c=(-2,-2)$. Now choose a surface $\Sigma$ homologous to $\Delta$. Observe that, since $\Delta$ is a sphere, one can construct such surfaces $\Sigma$ of any genus by attaching nullhomologous handles to $\Delta$. Then, for any almost complex structure $J$ on $X$, we have $c_{1}(J) \cdot \Sigma= \pm 4$ and $\Sigma \cdot \Sigma=2$. If we choose $\Sigma$ to have genus 0 or 4 , we see that there is an almost complex structure on $X$ that turns $\Sigma$ into a pseudoholomorphic curve, but if we choose a surface $\Sigma \sim \Delta$ with genus 1 , then there is no almost complex structure on $X$ such that $\Sigma$ is pseudoholomorphic. But on the other hand, the divisibility of $\Sigma$ clearly is one, so the equality $g(\Sigma) \equiv k(\Sigma) \bmod d$ is fulfilled for every value of $g(\Sigma)$.

Now consider the case $X=\mathbb{C} P^{2}$. Clearly, only the classes 3 and -3 occur as Chern classes of almost complex structures on $X$. If we construct a surface $\Sigma$ of genus one, representing the generator of $H^{2}(X ; \mathbb{Z})$, by attaching a handle to $\mathbb{C} P^{1}$, then, for any almost complex structure $J, c_{1}(J) \cdot \Sigma= \pm 3, \Sigma \cdot \Sigma=1$, so the adjunction equality will not hold for $J$. Again, we have $d=1$, and this provides the required example. 
Now we turn to the case $X=\mathbb{C} P^{2} \# \overline{\mathbb{C} P^{2}}$. Let $\Sigma$ be a surface of genus one, representing the class $(1,0) \in H^{2}(X ; \mathbb{Z})=\mathbb{Z} \times \mathbb{Z}$. Then the divisibility is 1 , and we have to prove that there is no almost complex structure such that $\Sigma$ is pseudoholomorphic. If $J$ is an almost complex structure on $X$ with first Chern class $c=(x, y)$, then $x^{2}-y^{2}=8$. If $\Sigma$ would be pseudoholomorphic with respect to $J$, this would imply $c \cdot \Sigma=x=1$; hence $1-y^{2}=8$, a contradiction. This proves that there is no such $J$.

Finally, to settle the case $X=\mathbb{C} P^{2} \# k \overline{\mathbb{C} P^{2}}$ and $k \geq 2$, note that $X$ is diffeomorphic to $\left(S^{2} \times S^{2}\right) \#(k-1) \overline{\mathbb{C} P^{2}}([\mathrm{~W} 2])$. Again let $\Delta \subset X$ denote the sphere coming from the diagonal embedding in $S^{2} \times S^{2}$, choose a surface $\Sigma$ representing the same homology class and let $g$ denote its genus. With respect to the basis of $H^{2}(X ; \mathbb{Z})$ coming from a diffeomorphism $X \cong\left(S^{2} \times S^{2}\right) \#(k-1) \overline{\mathbb{C} P^{2}}$, we have $[\Sigma]=(1,1,0, \ldots, 0)$, and if $J$ is an almost complex structure with Chern class $c=(x, y, a)$, with $a \in H^{2}\left((k-1) \overline{\mathbb{C} P^{2}}\right)$ and $x, y \in \mathbb{Z}$, then $c^{2}=2 x y+a \cdot a=9-k$ (note that $a \cdot a \leq 0$, here the dot denotes the cup product in the cohomology of $\left.(k-1) \overline{\mathbb{C} P^{2}}\right)$. Now $a \cdot a \leq-k+1$, since $a \equiv w_{2}\left((k-1) \overline{\mathbb{C} P^{2}}\right) \bmod 2$ and the intersection form of $(k-1) \overline{\mathbb{C} P^{2}}$ is standard, and we can conclude

$$
2 x y=9-k-a \cdot a \geq 8 .
$$

Therefore, as in the example $X=S^{2} \times S^{2}$, we have $x y \geq 4$, and $x, y \equiv 0 \bmod 2$. Now suppose that $\Sigma$ is pseudoholomorphic with respect to $J$. Then we have the adjunction equality $c \cdot \Sigma=x+y=4-2 g$. Together with $x y \geq 4$, this implies $g=0$ or $g \geq 4$. But we can construct $\Sigma$ by attaching one handle at $\Delta$ and therefore realize $g=1$. Hence this surface is not pseudoholomorphic with respect to any almost complex structure on $X$, and this completes the proof.

Note that the last part of the proof can be applied to every $X$ of the type $\left(S^{2} \times S^{2}\right) \# N$, where $N$ has no 2-torsion in its homology and negative definite intersection form (which must be standard, according to Donaldson).

Proof of Proposition 2. The intersection form $Q$ can be considered as a non-degenerate symmetric bilinear form on the real cohomology $H^{2}(X ; \mathbb{R})$, where the free part of the integral cohomology is lying as a lattice in this real vector space. Choose a class $\gamma \in H^{2}(X ; \mathbb{Z})$ with self-intersection $s=\gamma \cdot \gamma \neq 0$ and divisibility one. First suppose that $Q$ is positive definite. Then, for every class $c \in H^{2}(X ; \mathbb{Z})$, we have the Cauchy-Schwarz inequality $|Q(\gamma, c)|^{2} \leq s Q(c, c)$. If $c$ is the Chern class of an almost complex structure $J$, this implies $|Q(\gamma, c)|^{2} \leq s\left(2 \chi+3 b_{2}\right)$. If $\Sigma$ is a representative of $\gamma$ that is pseudoholomorphic with respect to $J$, we therefore have $(2-2 g+s)^{2} \leq$ $s\left(2 \chi+3 b_{2}\right)$. Note that the number on the right side of this inequality must be non-negative, otherwise there is no almost complex structure on $X$ at all. Hence we see that there is an upper bound for the genus of pseudoholomorphic curves representing $\gamma$ that does not depend on $J$; therefore a representative with large genus provides the required example. A similar argument works if $Q$ is negative definite.

Proof of Proposition 3. If $Q$ is definite, then the assertion of the proposition is covered by Proposition 2 If both $b^{+}$and $b^{-}$equal 1, then $Q$ must be the intersection form of $S^{2} \times S^{2}$ or of $\mathbb{C} P^{2} \# \overline{\mathbb{C} P^{2}}$; hence the intersection form of a rational surface. The same is true if $b^{+}=1$ and $Q$ is odd, all these cases are covered by Proposition 1 So the last case that is not covered by any of the preceding examples is the case 
$b^{-}=1$ and $b^{+} \geq 2$. In this case, choose a 4-manifold $X^{\prime}$ with intersection form $Q$. Let $h\left(X^{\prime}\right)=2 \chi\left(X^{\prime}\right)+3 \tau\left(X^{\prime}\right)$. Choose a class $\gamma$ in $H^{2}\left(X^{\prime} ; \mathbb{Z}\right)$ with divisibility 1 and $-s^{2}:=Q(\gamma, \gamma)<0$. Consider the lattice defined by the integral cohomology in the semi-euclidean vector space $H^{2}(X ; \mathbb{R})$. Choose a basis $e_{1}, \ldots, e_{n}$ of this vector space such that with respect to this basis, $Q$ is given by the matrix

$$
Q=\left(\begin{array}{cccccc}
-1 & & & & \\
& 1 & & & & \\
& & 1 & & \\
& & & \cdot & & \\
& & & & \cdot & \\
& & & & & 1
\end{array}\right)
$$

where all other entries are zero, and such that $\gamma=s e_{1}$ (more precisely, the free part of $\gamma$ ). Now choose a surface $\Sigma$ representing $\gamma$ with arbitrary genus $g$. Let $X=X^{\prime} \# k\left(S^{1} \times S^{3}\right)$, where $k$ is chosen large, such that $h(X)=2 \chi(X)+3 \tau(X)<$ $-\left(\frac{2-2 g}{-s}+s\right)^{2}$ and $b_{1}(X)+b_{2}^{+}(X) \equiv 1 \bmod 2$ (note that attaching a copy of $S^{1} \times S^{3}$ decreases the Euler characteristic $\chi(X)$ by 2 without changing the second cohomology and the intersection form). Now if $J$ would be any almost complex structure on $X$ such that $\Sigma$ is pseudoholomorphic with respect to $J$, and $c=\sum_{i} c_{i} e_{i} \in H^{2}(X ; \mathbb{R})$ its (real) first Chern class, then the adjunction equality would imply $c \cdot \Sigma=-s c_{1}=$ $2-2 g-s^{2}$; hence

$$
Q(c, c)=-c_{1}^{2}+\sum_{i \neq 1} c_{i}^{2} \geq-c_{1}^{2}=-\left(\frac{2-2 g}{-s}+s\right)^{2}>h(X)
$$

in contradiction to $c^{2}=2 \chi(X)+3 \tau(X)=h(X)$. This proves that $\Sigma$ cannot be pseudoholomorphic for any $J$, although, since the divisibility of $\gamma$ is one, the condition $g \equiv k(\gamma) \bmod d$ is fulfilled.

Finally I would like to thank my advisor, Dieter Kotschick, for his support and the helpfull discussions during the work on this paper.

\section{REFERENCES}

[D] S.K. Donaldson, Connections, cohomology and the intersection form of 4-manifolds, J. Differential Geom. 24 (1986), 275-342. MR 88g:57033

$[\mathrm{HH}]$ F. Hirzebruch, H. Hopf, Felder von Flächenelementen in 4-dimensionalen Mannigfaltigkeiten, Math. Ann. 136, (1958) 156-172. MR 20:7272

[Fu] M. Furuta, Monopole equation and the 11/8-conjecture, Preprint, 1995.

$[\mathrm{MH}]$ J. Milnor, D. Husemoller, Symmetric bilinear forms, Springer-Verlag, 1973. MR 58:22129

[Mi] G. Mikhalkin, J-holomorphic curves do not always minimize the genus, Proc. Amer. Math. Soc, 125 (1997), 1831-1833. MR 97g:57047]

[W1] C.T.C. Wall, On the orthogonal groups of unimodular quadratic forms, Math. Ann. 147 (1962), 328-338. MR 25:2009

[W2] C.T.C. Wall, Diffeomorphisms of 4-manifolds, J. London Math. Soc. 39 (1964), 131-140. MR 29:626

[W] H. Whitney, On the topology of differentiable manifolds, Lectures in Topology, Ann Arbor, Michigan, 1941. MR 3:133a

Mathematisches Institut der LMU, Theresienstr. 39, 80333 München, Germany

E-mail address: bohr@rz.mathematik.uni-muenchen.de 